\title{
Self refractive nonlinearities in chalcogenide based glasses
}

\author{
J. Requejo-Isidro ${ }^{\text {a) }}$, A.K. Mairaj ${ }^{\text {b) }}$, V. Pruneric), D.W. Hewak ${ }^{\text {b) }}$, \\ M.C. Nettid ${ }^{\text {d) J.J. Baumberg }}{ }^{\text {d) }}$
}

a) Instituto de Óptica, CSIC. Serrano, 121. 28006 Madrid. Spain.

b) Optoelectronics Research Centre, University of Southampton, SO17 1BJ, UK.

c) Optoelectronics Research Centre. Presently at Corning-OTI, Viale Sarca 222, Milano, Italy

d) Department of Physics \& Astronomy, University of Southampton, SO17 1BJ, UK.

\begin{abstract}
We report third order nonlinear absorption and refraction measurements at 1.20 $\mu \mathrm{m}$ and $1.52 \mu \mathrm{m}$ on selected gallium lanthanide sulfide-based glasses (Ga:La:S) showing negligible nonlinear absorption and a refractive nonlinearity close to one hundred times that of $\mathrm{SiO}_{2}$. Their potential use in telecommunication as base materials for all-optical switching practical devices is evaluated. The addition of a glass modifier to the Ga:La:S matrix has improved thermal and optical properties, resulting in ease of fibre drawing. The nonlinear optical response of this new variant of the Ga:La:S family is studied.
\end{abstract}

PACS numbers: 42.65.-k, 42.70.Ce, 42.70.Km, 78.20.Ci

Keywords: Nonlinear Optics, Glasses, Infrared transmitting materials, Optical constants. 
Third order nonlinear optical properties are of major interest in the development of optical devices. Although many materials have been explored as candidates for alloptical switching (AOS) devices, third order nonlinear measurements have been mainly performed at visible wavelengths. There are but few examples of third order nonlinear measurements at wavelengths of interest in optical communications.

Any nonlinear material suitable for an all-optical switching process should show a large third order optical nonlinearity, fast response time, and minimal linear and nonlinear absorption in the wavelengths of interest. Otherwise, a large absorbed power in a small volume would result in slow thermal drift in the refractive index. Furthermore, two-photon absorption (TPA) limits the optical power that can be transmitted and hence the attainable nonlinear phase shift in all-optical switching ${ }^{1}$. Assuming negligible linear absorption, it is customary to define the following figure of merit (FOM), $F O M=n_{2} /(\beta \cdot \lambda)$, where $\lambda$ is the wavelength, $n_{2}$ is the real part of the nonlinear refractive index and $\beta$ the TPA coefficient. For practical devices, this FOM should be much larger than 1 to avoid large TPA-induced attenuation.

The family of chalcogenide glasses shows a large intensity dependent index of refraction without appreciable linear absorption at wavelengths for optical communications. Some chalcogenide glasses have already been demonstrated as effective all-optical switching devices ${ }^{2}$. Chalcogenides based on gallium-sulphides are of great interest particularly due to their low toxicity, high transition temperature $\left(T_{g}=560^{\circ} \mathrm{C}\right)$ and excellent rare-earth solubility ${ }^{3}$. Improving the inherent properties of a glass is possible through the addition of glass modifiers. J. Wang et al. investigated the addition of $\mathrm{CsCl}$ to the Ga:La:S glass matrix with resulting improvements in optical properties and ease of fiber drawing of this modified glass ${ }^{4}$. 
We report nonlinear absorption and nonlinear refractive index measurements for selected variants of the Ga:La:S glass system. The measurements were at wavelengths of interest for optical communications. The selection of different glass compositions studied has been based on their viability to be drawn into fiber.

Most glasses based on the Ga:La:S system are fabricated in similar fashion. GLS glass (with molar composition $65 \mathrm{Ga}_{2} \mathrm{~S}_{3}: 32 \mathrm{La}_{2} \mathrm{~S}_{3}: 3 \mathrm{La}_{2} \mathrm{O}_{3}$ ) is fabricated by melting a mixture of gallium sulphide, lanthanum sulphide and lanthanum oxide powders. Batches of powders are placed in a vitreous carbon crucible and melted in a tube furnace at $1150^{\circ} \mathrm{C}$ for up to $24 \mathrm{~h}$. The tube furnace has an argon-purged atmosphere, facilitating the removal of volatile impurities, particularly $\mathrm{OH}$. The melt is quenched and the glass is annealed. Annealing is routinely done at $530^{\circ} \mathrm{C}$ (below glass transition temperature) for about $24 \mathrm{~h}$. The process of annealing removes residual stresses and thermal shock in the glass, which is introduced during the quench, making the glass suitable to be cut and polished.

The substitution of up to $30 \mathrm{~mol} \%$ of $\mathrm{La}_{2} \mathrm{~S}_{3}$ by $\mathrm{La}_{2} \mathrm{O}_{3}$, (with resulting molar composition $70 \mathrm{Ga}_{2} \mathrm{~S}_{3}: 30 \mathrm{La}_{2} \mathrm{O}_{3}$ ) results in a variant of GLS termed oxide-GLS (GLSO). GLSO glass has improved thermal stability during fiber drawing as compared to GLS; however, the higher oxide content in GLSO glass lowers the refractive index and deteriorates its spectroscopic properties ${ }^{5}$.

The addition of a modifier such as a lanthanum halide to the Ga:La:S glass matrix has been successfully demonstrated and provides several important properties over GLS and GLSO glasses. Halide doped Ga:La:S glass (GLSOF) (molar composition $70 \mathrm{Ga}_{2} \mathrm{~S}_{3}: \mathrm{xLaF}_{3}: 30-\mathrm{xLa}_{2} \mathrm{O}_{3}$ ) contains significantly less lanthanum oxide as 
compared to GLSO. This reduction in oxide content results in lower loss fiber due to reduction of hydroxyl impurities, while still maintaining thermal stability for fiber drawing ${ }^{4}, 6$

The transmittances of the materials were measured in order to determine the optical absorption edge (or optical gap), $\varepsilon_{g}$, as well as the linear refractive indices at $1.20 \mu \mathrm{m}$ and $1.52 \mu \mathrm{m}$ (Typical transmittance spectra of the samples under study are shown in Fig. 1, together with SF59 for reference purposes). Chalcogenide glasses, like almost all amorphous materials, exhibit an optical absorption edge which is not sharp, but obeys an exponential dependence on photon energy. Beyond the optical gap, the absorption, $\alpha$, changes its behavior and becomes $\alpha(\omega) \propto\left(\hbar \omega-\varepsilon_{g}\right)^{2} / \omega^{7}$. Thus, the value of the absorption edge at room temperature (shown in Table I) was obtained by linearly extrapolating $(\omega \alpha(\omega))^{\frac{1}{2}}$ versus $\hbar \omega$.

The linear refraction indices (shown in Table I) were computed with data from the transmittance spectra. The measured values for GLS, GLSO ${ }^{8}$ and SF59 (Schott catalogue) glasses were compared with those already published, showing a maximum deviation of $1.6 \%$.

Nonlinear measurements were carried out using the Z-scan technique 9 . In this experiment, the transmittance of the material is recorded in the far field through a small aperture as the sample is moved along the propagation path of a focused Gaussian laser beam. The transformation of phase distortion into amplitude distortion during beam propagation allows measurement of change in refractive index, $\Delta n=n_{2} \cdot I$, where $n_{2}$ is the nonlinear refractive index and $I=\left(n_{o} c / 8 \pi\right) \cdot \mid E(\omega)^{2}$ the time-averaged intensity of 
the optical field $E(\omega), n_{0}$ the linear refractive index and $c$ the speed of light ${ }^{10}$. The determination of the nonlinear absorption coefficient was carried out through the so called open aperture (or near field) configuration for Z-scan as described in reference ${ }^{11}$.

By fitting the Z-scan data with a thin lens model ${ }^{12}$ (provided that the thin lens condition is met, i.e. the thickness of the sample is much smaller than the Rayleigh range of the beam) it is possible to extract values for the nonlinear refractive index, $n_{2}$, the thermo-optic coefficient, and the two-photon absorption coefficient, $\beta$. An actual far field Z-scan fit for one of the samples under study is shown in Fig. 2. Pure electronic effects and thermo-optic effects can be differentiated because of the distinctive $z$ dependence of the normalized power transmitted by the sample.

The laser source used in the Z-scan experiment was an optical parametric amplifier pumped by a regeneratively amplified femtosecond Ti:Sapphire laser. The output of the system was a linearly polarized pulsed beam with a repetition rate of 230 $\mathrm{kHz}$. At the working wavelengths $(1.20 \mu \mathrm{m}$ and $1.52 \mu \mathrm{m})$, the pulse duration was typically $130 \mathrm{fs}$ and the power incident on the sample $\sim 200 \mathrm{~kW}$. The conditions of the nonlinear measurements were chosen to keep any thermal contribution to a minimum.

Uncertainties in the experimental data arise when determining the intensity at the sample. To reduce them, a standard SF59 sample was used as a reference, with a published value ${ }^{13}$ of $n_{2}=3.20 \times 10^{-15} \mathrm{~cm}^{2} / \mathrm{W}$ at $\lambda=1.06 \mu \mathrm{m}$. Since this (and the wavelengths we worked at) are far beyond the energy gap of SF59 (Fig. 1) it can be assumed that the nonlinear dispersion in the range of wavelengths of interest is negligible. Hence, we considered that the value of the nonlinear refractive index for SF59 at $1.06 \mu \mathrm{m}$ holds for $1.20 \mu \mathrm{m}$ and $1.52 \mu \mathrm{m}$ and used it as reference. This 
assumption was proved correct after cross-checking our GLS nonlinear measurements with those already published: comparison between measured and published data for GLS glass showed little variation ${ }^{14,15}$. Typical intensities inside the sample were around $2 \mathrm{GW} / \mathrm{cm}^{2}$ and no optical damage was observed.

Nonlinear refractive index measurements at $1.52 \mu \mathrm{m}$ for Ga:La:S based glasses are summarized in Table II. Measurements at $1.20 \mu \mathrm{m}$ and $1.52 \mu \mathrm{m}$ yielded the same value, within experimental resolution of $2 \%$, confirming the assumed flatness of nonlinear dispersion. Nonlinear absorption (multiple photon absorption) measurements were carried out in search of both two and three photon effects; no two-photon absorption was expected as the band gaps of all the samples are below $2.64 \mathrm{eV}$. (470 $\mathrm{nm}$, which is less than half the shortest wavelength used in the measurements). However, three photon absorption could become significant near $\varepsilon_{g} / 3$. No nonlinear absorption, regardless of its origin, could be resolved in a $2 \%$ (maximum experimental resolution, Fig 2.) full scale open aperture Z-scan trace. We therefore estimate $\beta<0.01$ $\mathrm{cm} / \mathrm{GW}$ at both $1.20 \mu \mathrm{m}$ and $1.52 \mu \mathrm{m}$ for all the samples.

The refractive nonlinearity results for Ga:La:S glasses show values close to a hundred times that of silica glasses. This demonstrates Ga:La:S glasses have larger offresonant nonlinearities than oxide and heavy metal oxide glasses ${ }^{14,16}$, however they are not the highest nonlinear chalcogenide glass (Table II). On the other hand, the small TPA of Ga:La:S glasses is one of their most outstanding features. When considering practical all-optical switching devices, both the nonlinear refractive index and the twophoton absorption coefficient must be taken into account. 
The large off-resonance nonlinear refractive index of $\mathrm{Ga}: \mathrm{La}: \mathrm{S}$ glasses is attributed to the large hyperpolarizability of the sulfide anions and cations with filled outer $d$ shells $\left(\mathrm{Ga}^{3+}\right)^{17}$. The non-linearity of the similar structure $70 \mathrm{Ga}_{2} \mathrm{~S}_{3} \times \mathrm{xa}_{2} \mathrm{~S}_{3} \mathrm{yLa}_{2} \mathrm{O}_{3}(30-\mathrm{x}-\mathrm{y}) \mathrm{LaF}_{3}$ glasses increases with increasing contents of sulfides as the sulfide anion hyperpolarizability is larger than that of the $\mathrm{O}^{2-}$ anion which in turn is larger than that of the fluorine anion.

The fact that the positive nonlinear refractive indices of the Ga:La:S glasses are predominantly due to bound electronic effects ${ }^{14}$, and the small TPA showed by these glasses account for the absence of measurable nonlinear dispersion at the working wavelengths, as the process responsible for TPA also gives a significant contribution to $n_{2}^{18}$

Table II lists the nonlinear optical refractive indices and absorption coefficients for the glasses studied in this work. Measurements made on Ga:La:S glasses are compared with those of the highest nonlinear chalcogenide, to our best knowledge, glasses (as reported in literature). Although $n_{2}$ values for Ga:La:S glasses are 5 to 7 times smaller than those of other chalcogenide glasses, the nonlinear absorption values for 'Ga:La:S glasses are much smaller. This leads to a figure of merit for Ga:La:S glasses (defined as above) much larger than 1 and among the highest in the family of chalcogenide glasses (Table II). The improved fiber drawing ability of GLSOF glasses should allow the realization of low loss single mode fiber and, although FOM for GLSOF glasses is slightly lower as compared with GLS, this is an acceptable tradeoff. Thus, GLSOF glass is a promising candidate for practical all-optical switching devices with fiber-based geometry. 
In summary, the large nonlinearity of Ga:La:S glasses with negligible nonlinear absorption at the telecommunication wavelengths indicate great potential for all-optical switching devices. Furthermore, material optimization of Ga:La:S glass with the addition of a third component results in a trade-off between nonlinear optical property versus glass workability. This shows that Ga:La:S based glasses are among the best candidates for practical photonic applications in the form of fibers.

\section{Acknowledgements}

This work has been partially supported by a British Council/CSIC 99-00 scholarship awarded to the first author and also support by HEFCE JR98SOBA. We thank Dr Eleanor Tarbox for helpful discussions. 


\section{References}

1 V. Mizrahi, K. W. DeLong, G. I. Stegeman, M. A. Saifi, and M. J. Andrejco, Optics Letters 14, 708-715 (1989).

2 M. Asobe, H. Kobayashi, and H. Itoh, Optics Letters 18, 1056-1058 (1993).

3 D. W. Hewak, R. C. Moore, T. Schweizer, J. Wang, B. Samson, W. S. Brocklesby, D. N. Payne, and E. J. Tarbox, Electronics Letters 32, 384-385 (1996).

4 J. Wang, J. R. Hector, D. Brady, D. Hewak, B. Brocklesby, M. Kluth, R. Moore, and D. N. Payne, Applied Physics Letters 71, 1753-1755 (1997).

5 D. W. Hewak, J. A. Medeiros Neto, B. N. Samson, R. S. Brown, K. P. Jedrzejewski, J. Wang, E. R. Taylor, R. I. Laming, G. Wylangowski, and D. N. Payne, IEEE Photonics Technology Letters 6, 609-612 (1994).

A. K. Mairaj, M. N. Petrovich, Y. W. West, A. Fu, D. W. J. Harwood, L. N. Ng,

T. M. Monro, N. G. Broderick, and D. W. Hewak, in Advances in Gallium Lanthanum Sulphide Glass for Optical Fibre and Devices. Photonics East, Boston, Masachusets, 2000.

7 S. R. Elliott, Physics of Amorphous Materials, 2 ed. (Longman Scientific \& Technical, Harlow, 1990).

8 H. Yayama, S. Fujino, K. Morinaga, H. Takebe, D. W. Hewak, and D. N. Payne, Journal of Non-Crystalline Solids 239, 187-191 (1998).

M. Sheik-Bahae, A. A. Said, and E. W. Van Stryland, Optics Letters 14, 955957 (1989).

W. L. Smith, in CRC Handbook of Laser Science and Technology; Vol. III, Part 1, edited by M. J. Weber (CRC Press, Inc., Boca Raton, Florida, 1986), p. 259281.

M. Sheik-Bahae, A. A. Said, T. Wei, D. J. Hagan, and E. W. Van Stryland, IEEE Journal of Quantum Electronics 26, 760-769 (1990).

R. F. Haglund Jr., in Handbook of Optical Properties; Vol. II. Optics of Small Particles, Interfaces and Surfaces, 1 ed., edited by R. E. Hummel and P. Wißmann (CRC Press, Inc., Boca Raton (Florida), 1997), p. 191-231. Weidman, Applied Physics Letters 54, 1293-1295 (1989). 
S. Smolorz, I. Kang, F. Wise, B. G. Aitken, and N. F. Borelli, Journal of NonCrystalline Solids 256-257, 310-317 (1999).

15 I. Kang, T. D. Krauss, F. Wise, B. G. Aitken, and N. F. Borelli, Journal of the Optical Society of America B 12, 2053-2059 (1995).

16 N. F. Borelli, B. G. Aitken, M. A. Newhouse, and D. W. Hall, Journal of Applied Physics 70, 2774-2779 (1991).

17 R. Adair, L. L. Chase, and S. A. Payne, Physical Review B 39, 3337-3350 (1989).

18 M. Sheik-Bahae, D. J. Hagan, and E. W. Van Stryland, Physical Review Letters 65, 96-99 (1990).

19 F. Smektala, C. Quemard, V. Couderc, and A. Barthélémy, Journal of NonCrystalline Solids 274, 232-237 (2000).

20 G. Lenz, J. Zimmermann, T. Katsufuji, M. E. Lines, H. Y. Hwang, S. Spälter, R. E. Slusher, S. W. Cheong, J. S. Sanghera, and I. D. Aggarwal, Optics Letters 25, 254-256 (2000).

21 R. Rangel-Rojo, T. Kosa, E. Hajto, P. J. S. Ewen, A. E. Owen, A. K. Kar, and B.

S. Wherrett, Optics Communications 109, 145-150 (1994). 
Tables and figures.

Table I

\begin{tabular}{lcccccccc} 
& \multicolumn{3}{c}{ Molar Composition (mol \%) } & \multicolumn{3}{c}{ Refractive index Absorption edge (nm) } \\
& $\mathrm{Ga}_{2} \mathrm{~S}_{3}$ & $\mathrm{Na}_{2} \mathrm{~S}$ & $\mathrm{La}_{2} \mathrm{~S}_{3}$ & $\mathrm{La}_{2} \mathrm{O}_{3}$ & $\mathrm{LaF}_{3}$ & $1.20 \mu \mathrm{m}$ & $\frac{1.52 \mu \mathrm{m}}{1.52}$ & \\
GNS & 68 & 32 & & & & 2.16 & 2.14 & 473 \\
GLS & 65 & & 32 & 3 & & 2.42 & 2.41 & 545 \\
GLSO & 70 & & & 30 & & 2.25 & 2.25 & 500 \\
GLSOF & 70 & & & 15 & 15 & 2.28 & 2.26 & 497
\end{tabular}


Table II

\begin{tabular}{|c|c|c|c|c|c|c|c|}
\hline & $\begin{array}{c}\lambda_{\text {meas }}{ }^{\mathrm{a}} \\
(\mu \mathrm{m})\end{array}$ & $n_{0}$ & $\begin{array}{c}n_{2} \\
\left(10^{-14} \mathrm{~cm}^{2} / \mathrm{w}\right)\end{array}$ & $\beta(\mathrm{cm} / \mathrm{GW})$ & FOM & $\begin{array}{l}\text { Absorption } \\
\text { edge }(\mathrm{nm})\end{array}$ & Author \\
\hline $\mathrm{Ge}_{10} A s_{10} \mathrm{Se}_{80}$ & 1.06 & $=$ & 22 & 2.7 & 0.77 & 760 & Smektala et $a l^{b}$ \\
\hline $\mathrm{Ge}_{30} \mathrm{Se}_{70}$ & 1.06 & - & 21 & 1.1 & 1.8 & 705 & Smektala et al. ${ }^{\mathrm{b}}$ \\
\hline $\mathrm{Ge}_{0.28} \mathrm{Se}_{0.60} \mathrm{Sb}_{0.12}$ & 1.5 & 2.6 & 9.4 & 0.2 & 3 & 690 & Lenz et al. ${ }^{c}$ \\
\hline $\mathrm{As}_{2} \mathrm{Se}_{3}$ & 1.5 & 2.8 & 13.0 & 0.4 & 2 & 700 & $\begin{array}{l}\text { Lenz et al. }^{c} \\
\text { Rangel-Rojo et }\end{array}$ \\
\hline $\mathrm{As}_{2} \mathrm{~S}_{3}$ & 1.06 & 2.6 & 5.7 & 2.6 & 2.1 & 520 & al. ${ }^{d}$ \\
\hline GNS & 1.52 & 2.14 & 1.01 & $<0.01$ & 6.6 & 473 & This work \\
\hline GLS & 1.52 & 2.41 & 2.16 & $<0.01$ & 14.2 & 545 & This work \\
\hline GLSO & 1.52 & 2.25 & 1.77 & $<0.01$ & 11.6 & 500 & This work \\
\hline GLSOF & 1.52 & 2.26 & 1.39 & $<0.01$ & 9.1 & 497 & This work \\
\hline
\end{tabular}

${ }^{a}$ Wavelength used for each measurement.

${ }^{b}$ Reference ${ }^{19}$.

${ }^{c}$ Reference ${ }^{20}$.

${ }^{d}$ Reference ${ }^{21}$. 


\section{Figure Captions}

Fig. 1 Transmission spectra for samples under study. From $650 \mathrm{~nm}$ to $1800 \mathrm{~nm}$ the spectra are flat. Transmission spectra for GLSO and GLSOF samples are too close to be clearly resolved in the figure.

Fig. 2 Z-scan Near and far field (open and closed aperture configuration, respectively) trace for GLSO and actual far field data fit. 
Fig 1: Transmission spectra

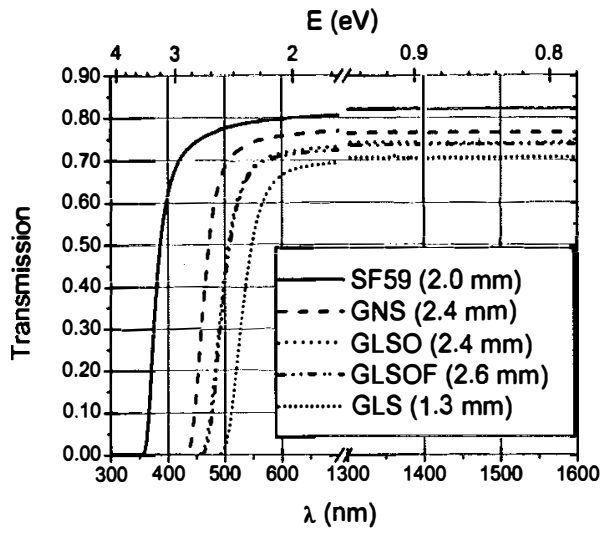


Fig. 2: Z-scan Near and far field (open and closed aperture configuration, respectively) trace for GLSO and actual far field data fit.

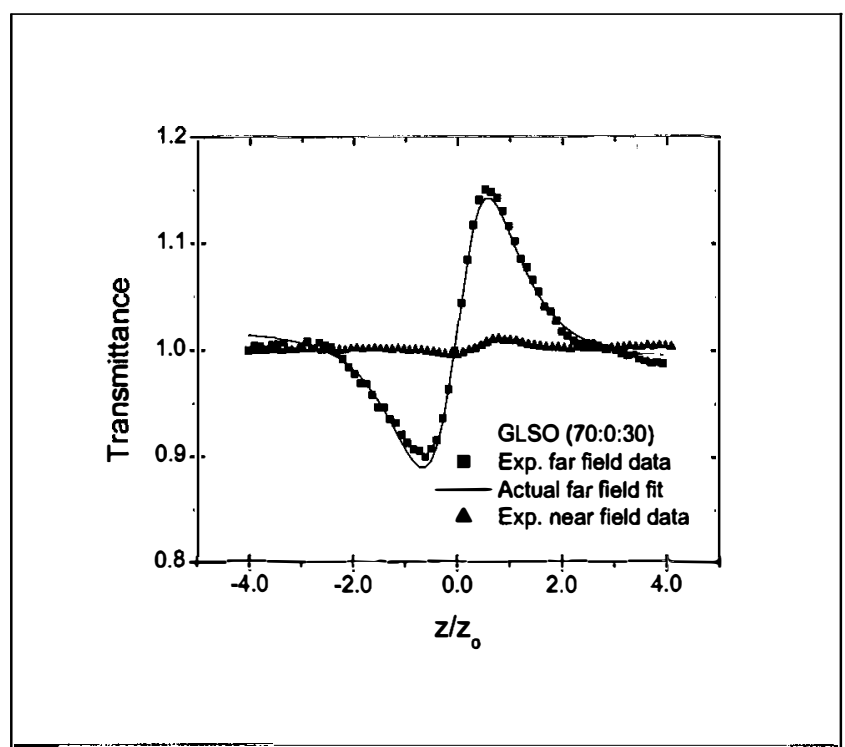

